\title{
A Higman-Haemers Inequality for Thick Regular Near Polygons
}

AKIRA HIRAKI

hiraki@cc.osaka-kyoiku.ac.jp

Division of Mathematical Sciences, Osaka Kyoiku University, Asahigaoka 4-698-1, Kashiwara,

Osaka 582-8582, Japan

JACK KOOLEN*

jhk@euclid.postech.ac.kr

Combinatorial and Computational Mathematics Center, Pohang University of Science and Technology,

San 31, Hyoja-dong, Namgu, Pohang 790-784, Korea

Received August 30, 2002; Revised February 4, 2003; Accepted October 2, 2003

Abstract. In this note we will generalize the Higman-Haemers inequalities for generalized polygons to thick regular near polygons.

Keywords: regular near polygon, distance-regular graph

\section{Introduction}

The reader is referred to the next section for the definitions.

Generalized $n$-gons of order $(s, t)$ were introduced by Tits in [12]. Although formally $n$ is unbounded, a famous theorem of Feit-G. Higman asserts that, apart from the ordinary polygons, finite examples can exist only for $n=3,4,6,8$ or 12 . (See [5] and [3, Theorem 6.5.1].)

If $s>1$ and $t>1$, then $n=12$ is not possible. Moreover in the case of $n=4,6,8$, D.G. Higman [8, 9] and Haemers [7] showed that $s$ and $t$ are bounded from above by functions in $t$ and $s$, respectively. To show this they used the Krein condition. (See also [3, Theorem 6.5.1].)

Let $\Gamma$ be a thick regular near $2 d$-gon of order $(s, t)$ and let $t_{i}:=c_{i}-1$ for all $1 \leq i \leq d$. Brouwer and Wilbrink [4] showed

$$
\sum_{i=0}^{d-1}\left(\frac{-1}{s^{2}}\right)^{i} \prod_{j=1}^{i}\left(\frac{t-t_{j}}{1+t_{j}}\right) \geq 0 .
$$

This was proved from the Krein condition $q_{d d}^{d} \geq 0$. If $d$ is even, then $1+t \leq\left(s^{2}+1\right)$ $\left(1+t_{d-1}\right)$.

A similar result was shown by Mathon for regular near hexagons.

*The author thanks the Com2MaC-KOSEF for its support. His current address is Div Appl Math, KAIST, YusongKu Deajon, Korea. 
In this note we are going to show that for thick regular near $2 d$-gons of order $(s, t), t$ is bounded from above by a function of $s$ and the diameter $d$.

In particular, we show the following results. We will only use the multiplicity of the smallest eigenvalue to show those results.

Theorem 1 Let $\Gamma$ be a distance-regular graph of order $(s, t)$ with $s>1$. Let $d$ be the diameter of $\Gamma, r:=\max \left\{i \mid\left(c_{i}, a_{i}, b_{i}\right)=\left(c_{1}, a_{1}, b_{1}\right)\right\}$ and $\rho:=\frac{d}{r}$. Suppose $-t-1$ is an eigenvalue of $\Gamma$. Then $t<s^{4 \rho-1}$.

Corollary 2 Let $\Gamma$ be a thick regular near $2 d$-gon of order $(s, t)$. Let $r:=\max \{i$ $\left.\left(c_{i}, a_{i}, b_{i}\right)=\left(c_{1}, a_{1}, b_{1}\right)\right\}$ and $\rho:=\frac{d}{r}$. Then the following hold.

(1) $t<s^{4 \rho-1}$.

(2) If $r \notin\{1,2,3,5\}$, then $t<s^{7}$.

A generalized $2 d$-gon of order $(s, t)$ is a regular near $2 d$-gon of order $(s, t)$ with $d=r+1$. It is known that if a generalized $2 d$-gon of order $(s, t)$ exists, then there exists a generalized $2 d$-gon of order $(t, s)$ which is known as dual. So as a consequence of this corollary we will show that for generalized $2 d$-gons we can bound $s$ and $t$ by functions in $t$ and $s$, respectively.

Let $\Gamma$ be a generalized $2 d$-gon of order $(s, t)$. Then the following hold.

(1) If $s>1$, then $t<s^{\frac{3 d+1}{d-1}}$.

(2) If $t>1$, then $s<t^{\frac{3 d+1}{d-1}}$.

The bound given by Higman [8, 9] and Haemers [7] can be proved without using the Krein condition although the bound proved here is a bit weaker.

Let us consider another consequence of Corollary 2. Suppose it is true that for given $s$ and $t$ there are only finitely many regular near $2 d$-gons of order $(s, t)$. Then for given $s^{\prime}>1$ there are only finitely many regular near $2 d$-gons of order $\left(s^{\prime}, t^{\prime}\right)$ with $r=\max \{i \mid$ $\left.\left(c_{i}, a_{i}, b_{i}\right)=\left(c_{1}, a_{1}, b_{1}\right)\right\} \geq 6$. Furthermore, for a regular near $2 d$-gons of order $\left(s^{\prime}, t^{\prime}\right)$ the diameter $d$ is bounded by a function in $s^{\prime}$.

\section{Definitions}

Let $\Gamma=(V \Gamma, E \Gamma)$ be a connected graph without loops or multiple edges. For vertices $x$ and $y$ in $\Gamma$ we denote by $\partial_{\Gamma}(x, y)$ the distance between $x$ and $y$ in $\Gamma$. For a vertex $x$ in $\Gamma$ and a set $L$ of vertices we define $\partial_{\Gamma}(x, L):=\min \left\{\partial_{\Gamma}(x, z) \mid z \in L\right\}$.

The diameter of $\Gamma$, denoted by $d$, is the maximal distance of two vertices in $\Gamma$. We denote by $\Gamma_{i}(x)$ the set of vertices which are at distance $i$ from $x$.

A connected graph $\Gamma$ with diameter $d$ is called distance-regular if there are numbers

$$
c_{i}(1 \leq i \leq d), \quad a_{i}(0 \leq i \leq d) \quad \text { and } \quad b_{i}(0 \leq i \leq d-1)
$$


such that for any two vertices $x$ and $y$ in $\Gamma$ at distance $i$ the sets

$$
\Gamma_{i-1}(x) \cap \Gamma_{1}(y), \quad \Gamma_{i}(x) \cap \Gamma_{1}(y) \quad \text { and } \quad \Gamma_{i+1}(x) \cap \Gamma_{1}(y)
$$

have cardinalities $c_{i}, a_{i}$ and $b_{i}$, respectively. Then $\Gamma$ is regular with valency $k:=b_{0}$.

Let $\Gamma$ be a distance-regular graph with diameter $d$. The array

$$
\iota(\Gamma)=\left\{\begin{array}{lllllll}
* & c_{1} & \cdots & c_{i} & \ldots & c_{d-1} & c_{d} \\
a_{0} & a_{1} & \cdots & a_{i} & \ldots & a_{d-1} & a_{d} \\
b_{0} & b_{1} & \ldots & b_{i} & \ldots & b_{d-1} & *
\end{array}\right\}
$$

is called the intersection array of $\Gamma$. Define $r=r(\Gamma):=\max \left\{i \mid\left(c_{i}, a_{i}, b_{i}\right)=\left(c_{1}, a_{1}, b_{1}\right)\right\}$. The numerical girth of $\Gamma$ is $2 r+2$ if $c_{r+1} \neq 1$ and $2 r+3$ if $c_{r+1}=1$.

By an eigenvalue of $\Gamma$ we will mean an eigenvalue of its adjacency matrix $A$. Its multiplicity is its multiplicity as eigenvalue of $A$. Define the polynomials $u_{i}(x)$ by

$$
\begin{aligned}
& u_{0}(x):=1, \quad u_{1}(x):=x / k, \quad \text { and } \\
& c_{i} u_{i-1}(x)+a_{i} u_{i}(x)+b_{i} u_{i+1}(x)=x u_{i}(x), \quad i=1,2, \ldots, d-1 .
\end{aligned}
$$

Let $k_{i}:=\left|\Gamma_{i}(x)\right|$ for all $0 \leq i \leq d$ which does not depend on the choice of $x$.

Let $\theta$ be an eigenvalue of $\Gamma$ with multiplicity $m$. It is well-known that

$$
m=\frac{|V \Gamma|}{\sum_{i=0}^{d} k_{i} u_{i}(\theta)^{2}} .
$$

For more information on distance-regular graphs we would like to refer to the books [1-3] and [6].

A graph $\Gamma$ is said to be of order $(s, t)$ if $\Gamma_{1}(x)$ is a disjoint union of $t+1$ cliques of size $s$ for every vertex $x$ in $\Gamma$. In this case, $\Gamma$ is a regular graph of valency $k=s(t+1)$ and every edge lies on a clique of size $s+1$. A clique of size $s+1$ is called a singular line of $\Gamma$.

A graph $\Gamma$ is called (the collinearity graph of ) a regular near $2 d$-gon of order $(s, t)$ if it is a distance-regular graph of order $(s, t)$ with diameter $d$ and $a_{i}=c_{i}(s-1)$ for all $1 \leq i \leq d$.

A regular near $2 d$-gon is called thick if $s>1$.

A generalized $2 d$-gon of order $(s, t)$ is a regular near $2 d$-gon of order $(s, t)$ with $d=r+1$.

More information on regular near $2 d$-gons and generalized $2 d$-gons will be found in [ 3 , Sections 6.4-6.6].

\section{Proof of the theorem}

In this section we prove our theorem. First we recall the following result. 
Proposition 3 [11, Proposition 3.3] Let $\Gamma$ be a distance-regular graph with valency $k$, numerical girth $g$ such that each edge lies in an $\left(a_{1}+2\right)$-clique. Let $h$ be a positive integer. Suppose $\theta=-\frac{k}{a_{1}+1}$ be an eigenvalue of $\Gamma$ with multiplicity $m$. Then the following hold. (1) If $g \geq 4 h$, then

$$
m \geq 1+\frac{k a_{1}}{a_{1}+1} \frac{b_{1}^{h}-1}{b_{1}-1} .
$$

(2) If $g \geq 4 h+2$, then

$$
m \geq \frac{1}{a_{1}+1}+\frac{a_{1}\left(a_{1}+2\right)}{a_{1}+1} \frac{b_{1}^{h+1}-1}{b_{1}-1} .
$$

Lemma 4 Let $\Gamma$ be a distance-regular graph of order $(s, t)$ with diameter $d$. Suppose $-t-1$ is an eigenvalue of $\Gamma$ with multiplicity $m$. Then for any integer $i$ with $0 \leq i \leq d$, the following hold.

(1) Let $C$ be a clique of size $s+1$ and $x \in V \Gamma$ with $\partial_{\Gamma}(x, C)=i$. Then

$$
\alpha_{i}:=\left|\left\{z \in C \mid \partial_{\Gamma}(x, z)=i\right\}\right|
$$

does not depend on the choice of $C$ and $x$. Furthermore, $\partial_{\Gamma}(x, C) \leq d-1$ for any vertex $x$ in $\Gamma$.

(2) There exists an integer $\gamma_{i}$ such that $c_{i}=\gamma_{i} \alpha_{i-1}$ and $b_{i}=\left(t+1-\gamma_{i}\right)\left(s+1-\alpha_{i}\right)$.

(3) Let $u_{j}:=u_{j}(-t-1)$ for all $0 \leq j \leq d$. Then for all $1 \leq j \leq d$ we have

$$
u_{j}=\left(\frac{-\alpha_{j-1}}{s+1-\alpha_{j-1}}\right) u_{j-1} .
$$

In particular,

$$
u_{i}^{2} \geq\left(\frac{1}{s}\right)^{2 i}
$$

(4) $m \leq s^{2 d}$ with equality if and only if $s=1$.

Proof: (1) See [4, Lemma 13.7.2].

(2) Let $x$ and $y$ be vertices in $\Gamma$ at distance $i$. Let $\gamma_{i}$ be the number of singular lines through $y$ at distance $i-1$ from $x$. Each such clique has $\alpha_{i-1}$ vertices which are at distance $i-1$ from $x$. Hence we have $c_{i}=\gamma_{i} \alpha_{i-1}$. There are $t+1-\gamma_{i}$ singular lines through $y$ at distance $i$ from $x$. Each such clique has $s+1-\alpha_{i}$ vertices which are at distance $i+1$ from $x$. Then we have $b_{i}=\left(t+1-\gamma_{i}\right)\left(s+1-\alpha_{i}\right)$.

(3) We prove the first assertion by induction on $j$. The case $j=1$ is true since $u_{0}=$ $1, u_{1}=-\frac{1}{s}$ and $\alpha_{0}=1$. 
Assume $1 \leq j \leq d-1$ and $\alpha_{j-1} u_{j-1}=-\left(s+1-\alpha_{j-1}\right) u_{j}$. Then we have

$$
\begin{aligned}
b_{j} u_{j+1}= & \left(-t-1-a_{j}\right) u_{j}-c_{j} u_{j-1} \\
= & \left\{-t-1-(t+1) s+c_{j}+b_{j}\right\} u_{j}+\gamma_{j}\left(s+1-\alpha_{j-1}\right) u_{j} \\
= & \left\{-(t+1)(s+1)+\gamma_{j} \alpha_{j-1}+\left(t+1-\gamma_{j}\right)\left(s+1-\alpha_{j}\right)\right. \\
& \left.+\gamma_{j}\left(s+1-\alpha_{j-1}\right)\right\} u_{j} \\
= & -\left(t+1-\gamma_{j}\right) \alpha_{j} u_{j}
\end{aligned}
$$

from (2). The first assertion is proved. Since

$$
\left(\frac{-\alpha_{j-1}}{s+1-\alpha_{j-1}}\right)^{2} \geq\left(\frac{1}{s}\right)^{2}
$$

the second assertion follows from the first assertion.

(4) We have

$$
M:=\sum_{i=0}^{d} k_{i} u_{i}^{2} \geq \sum_{i=0}^{d} k_{i}\left(\frac{1}{s}\right)^{2 i} \geq\left(\frac{1}{s}\right)^{2 d} \sum_{i=0}^{d} k_{i}=\frac{|V \Gamma|}{s^{2 d}} .
$$

Hence

$$
m=\frac{|V \Gamma|}{M} \leq s^{2 d}
$$

Proof of Theorem 1: We remark that $a_{1}=s-1$ and $b_{1}=s t$. Let $g$ be the numerical girth of $\Gamma$.

First we assume $r$ is odd with $r=2 h-1$. Then $g \geq 2 r+2=4 h$ and

$$
m>\frac{k a_{1}}{a_{1}+1} b_{1}^{h-1}=(t+1)(s-1)(s t)^{h-1}>s^{h-1} t^{h}
$$

from Proposition 3 (1). It follows, by Lemma 4 (4), that

$$
s^{(4 h-2) \rho}=s^{2 d} \geq m>s^{h-1} t^{h} .
$$

The desired result is proved.

Second we assume $r$ is even with $r=2 h$. Then $g \geq 2 r+2=4 h+2$ and $m>b_{1}^{h}$ from Proposition 3 (2). Hence we have

$$
s^{4 h \rho}=s^{2 d} \geq m>(s t)^{h} .
$$

The desired result is proved. 
In [10], we have shown the following result.

Proposition 5 Let $\Gamma$ be a thick regular near $2 d$-gon with $r=r(\Gamma)$. If $2 r+1 \leq d$ then for any integer $q$ with $r+1 \leq q \leq d-r$ there exists a regular near $2 q$-gon as a strongly closed subgraph in $\Gamma$. In particular, $r \in\{1,2,3,5\}$.

Proof of Corollary 2: It is known that a regular near $2 d$-gon of order $(s, t)$ has an eigenvalue $-t-1$. Moreover if $r \notin\{1,2,3,5\}$, then $d \leq 2 r$ from Proposition 5. Therefore the corollary is a direct consequence of Theorem 1 .

\section{References}

1. E. Bannai and T. Ito, Algebraic Combinatorics I: Association Schemes, Benjamin-Cummings Lecture Note Ser. 58, Benjamin/Cummings Publ. Co., London, 1984.

2. N.L. Biggs, Algebraic Graph Theory, Cambridge Tracts in Math. 67, Cambridge Univ. Press, 1974.

3. A.E. Brouwer, A.M. Cohen, and A. Neumaier, Distance-Regular Graphs, Springer, Heidelberg, 1989.

4. A.E. Brouwer and H.A. Wilbrink, "The structure of near polygons with quads," Geom. Dedicata 14 (1983) $145-176$.

5. W. Feit and G. Higman, "The non-existence of certain generalized polygons," J. Algebra 1 (1964), 114-131.

6. C.D. Godsil, Algebraic Combinatorics, Chapman and Hall, New York, 1993.

7. W.H. Haemers and C. Roos, "An inequality for generalized hexagons," Geom. Dedicata 10 (1981), $219-222$.

8. D.G. Higman, "Partial geometries generalized quadrangles and strongly regular graphs," pp. 263-293 in Atti del Convegno di Geometria, Combinatoria e sue Applicazioni (Univ. degli Studi di Perugia, Perugia, 1970), Perugia 1971.

9. D.G. Higman, "Invariant relations, coherent configurations and generalized polygons," pp. 27-43 in Combinatorics, Math Centre Tracts 57, Amsterdam, 1974.

10. A. Hiraki, "Strongly closed subgraphs in a regular thick near polygons," European J. Combin. 20 (1999), 789-796.

11. A. Hiraki and J.H. Koolen, "An improvement of the Godsil bound," Ann. of Combin. 6 (2002), $33-44$.

12. J. Tits, "Sur la trialité et certains groups qui s'en déduisent," Publ. Math. I.H.E.S. 2 (1959), 14-60. 